\title{
Effect of Direct Vibration on the Activity of Deep Trunk Muscles of Patients with Non-specific Chronic Low Back Pain
}

Nackhwan Kim ( $\sim$ nackhwan@gmail.com )

Korea University Medical Center

\section{Soo Hoon Yoon}

Korea University Medical Center

Minhee Kim

Korea University

Sang-heon Lee

Korea University Medical Center

\section{Dong Hee Kim}

Korea University Medical Center

Ki Hoon Kim

Korea University Medical Center

\section{Research Article}

Keywords: Spinal stabilization exercise, Vibration therapy, Muscle activity, Surface electromyography, Ultrasonography

Posted Date: March 31st, 2021

DOl: https://doi.org/10.21203/rs.3.rs-222429/v1

License: (9) This work is licensed under a Creative Commons Attribution 4.0 International License. Read Full License

Version of Record: A version of this preprint was published at Journal of Back and Musculoskeletal Rehabilitation on February 25th, 2022. See the published version at https://doi.org/10.3233/BMR210211. 


\section{Abstract}

Background: We used two methods, direct and indirect, to study the application of vibration training. This pilot study aimed to investigate the short-term effect of direct vibration and spinal stabilization exercises on the deep trunk muscles of patients with non-specific chronic low back pain (CLBP).

Methods: Sixty-two participants with non-specific CLBP were randomly placed into two groups, one with direct vibration to the trunk muscles, VSE (vibration-plus stabilization exercise) group, and one without, CSE (conventional stabilization exercise) group. The groups underwent twelve sessions of an exercise program for spinal stability. We measured the thickness and activity of the following trunk muscles using ultrasonography and surface electromyography (sEMG): transversus abdominis (TrA), external oblique (EO), internal oblique (IO), rectus abdominis (RA), lumbar multifidus (LM), and lumbar erector spinae (LES).

Results: The ultrasonographic examination revealed that the ratio of muscle thickness to muscle contraction and relaxation was increased after training in the TrA and LM muscles of patients in the CSE group, and in the TrA, LM, and IO muscles in the VSE group. We also found that the LM/LES ratio in the CSE group and the IO/RA, TrA/RA, and LM/LES ratios in the VSE group were significantly increased. Using multiple regression analysis, we showed that the improvement of the symptoms was related to increased activity of the deep trunk muscles in the VSE group $(p<0.05)$.

Conclusion: The findings suggest that direct vibration on the trunk muscles may increase the activity of contracting deep muscles and be used in addition to the effective enhancement of stabilization exercise in patients with non-specific CLBP.

Trial registration: KCT0003858. Registered 26 April 2019.

\section{Background}

Spinal stabilization training is a well-known therapeutic method commonly used in clinical practice today. The main goal of stabilization exercises is to improve the neuromuscular control, the strength, and the endurance of central muscles and maintain a dynamic spinal and trunk stability (1). Various studies with long-term outcomes showed that stabilization training as a single or a combined modality improved pain intensity and disability, and prevented the recurrence s of chronic low back pain (CLBP) episode $(2,3)$.

The application of physical training including vibrations has already been studied in the past. These studies were based on the improvement of neural adaptation and the reflex modification mechanism (4). Bosco et al. were the first to report an increase in leg strength when they analyzed the acute effect of vibration as an exercise modality on strength (5). Since then, there have been many reports about the positive impact of vibration on muscle strength and performance (6-8). However, little is known about the effect of vibration exercises on the clinical symptoms of patients with CLBP. One comparative study analyzed the impact of spinal stabilization exercises including whole-body vibration of $25 \mathrm{~Hz}$ for two 
weeks, and the authors did not find any significant difference in the endurance of the multifidus muscle with or without vibration (9).

Researchers used two different methods, indirect or direct, to apply vibration in vibration training studies. The method used to apply vibration can affect the intensity of the vibration load on the trained muscle as well as its magnitude, amplitude, and frequency (10). Nevertheless, no studies or clinical trials have been reported on the spinal stabilization with direct vibration on trunk muscles.

The purpose of this study is to investigate the short-term effect of direct vibration with spinal stabilization exercises on the deep trunk muscles of patients with non-specific chronic low back pain (CLBP).

\section{Methods}

\section{Design and participants}

To analyze the effect of direct vibration on trunk muscles, we assessed the maximal and rested contraction of trunk muscles after applying direct vibration during stabilization exercise. We measured the patients' anatomical and electrophysiological features using a randomized comparison study design. We measured contracted and rested muscles using ultrasound and surface electromyography. The participants performed preprogrammed spinal stabilization exercises and then scheduled three visits to measure the contraction of trunk muscles. The protocol was approved by the institutional review board of Korea University Anam Hospital in Korea. Each participant signed a written informed consent before participating in the study. All procedures were performed in accordance with relevant guidelines including a 'declaration of Helsinki'.

Participants were enrolled in this study based on the following inclusion criteria: non-specific CLBP for at least three months (11), and aged between 20 and 60 .

Participants were excluded if they displayed symptoms of recognizable pathologies such as infection, malignancy, inflammatory disease, structural deformity, overt neurologic signs, history of trauma, abdominal or lumbar surgery, and pregnancy (suspected or confirmed). The exclusion criteria from the clinical trial were: deterioration of back pain, severe physical or physiological damage, refusal of patient, drastic reduction of compliance, absence on three or more sessions, and any intervention of other treatments.

We used random allocation because the subjects' task or living environment could not be controlled. We computerized randomized assignments before enrollment. The subjects were enrolled in the order they were diagnosed with non-specific CLBP and approved for participation in this study.

Participants $(n=62)$ were randomly divided into two groups. Each group received a protocol including twelve sessions of treatment over four weeks and a follow-up visit 8 weeks after the end of the treatment. A flow chart describing the number of subjects considered for this clinical investigation is shown in Fig. 1. The study was conducted per the guidelines of the consolidated standards of reporting trials (CONSORT). 


\section{Intervention and device}

The spinal stabilization exercise protocol for the rehabilitation of CLBP patients is based on commonly advocated spinal stabilization exercises (12-14). The spinal stabilization exercise program consists of five different exercise types repeated in each session: upper-body extensions, alternate arm and leg lifts, alternate arm and leg extensions, diagonal curl-ups, and curl-ups. The subjects exercised three times a week in our gym under the supervision of experienced physical therapists. They helped the subjects to maintain accurate postures for 10-15 seconds and encouraged the completion of the 30 minutes exercise protocol, including warm-up, cool-down, and proper rests.

We designed and manufactured a vibration device $(330 \times 180 \times 120 \mathrm{~mm})$ for this study only. The target functions of the device were as follows: adjustable frequency from 0 to $100 \mathrm{~Hz}$, adjustable intensity (0$4 \mathrm{~mm}$, peak acceleration of 0-6g) in three steps, gradual start and end of vibration for compliance, exterior and materials designed to maintain contact on the body surface above the lumbar paraspinal muscles and increase the vibration transmission efficiency, interior structured to prevent loss of generated vibration, and immediate shutdown and removal during application.

\section{Outcomes}

We obtained ultrasonographic images of the rectus abdominis (RA), the external oblique (EO), the internal oblique (IO), the transversus abdominis (TrA) muscles, the lumbar erector spinae (LES), and the lumbar multifidus (LM) muscles at $\mathrm{L} 4 / 5$ level during both resting and activation states. We determined the patients' position and transducer location based on previous studies $(15,16)$. We measured the thickness of the contracted EO, IO, and TrA during an abdominal drawing-in maneuver (ADIM), of the RA during curlups, and of the LES and LM during maximal resistive lumbar extension on prone (17). We calculated the change in muscle thickness due to contraction for all muscles using the following equation: [thickness ratio $=$ thickness contracted $/$ thickness rest]. The transducer was vertically placed for the measures. We placed the inferior border immediately above the umbilicus and moved laterally from the midline. For the LES and LM muscles, we placed a curvilinear transducer longitudinally along the spine just lateral to L4 spinous process to identify the L4/5 zygapophyseal joint (12).

We recorded the muscle activity of the bilateral EO, IO, TrA, RA, LES, and LM muscles using surface EMG. We determined the patients' position and the testing protocol based on previous studies (18). We positioned electrodes on the abdominal muscles (EO, IO, TrA, and RA muscles) according to McGill (19). We placed the electrodes on the LES muscles by positioning them $4 \mathrm{~cm}$ laterally from the $L 4$ spinous process at the level of the iliac crest (20). For the LM muscles, we placed the electrodes above the posterior superior iliac spine on the spinous processes of the lumbar and sacral vertebrae $(21,22)$. We verified the quality of the EMG signal to ensure correct electrode placement (23), and used reference voluntary contractions (\%RVCs) to normalize muscle activation. We collected the raw EMG data using the wireless EMG system TeleMyo 2400R which was connected to a Noraxon Myosystem 1200 unit (Noraxon, Scottsdale, AZ, USA) with a sampling rate of $1000 \mathrm{~Hz}$. The mean activities of the muscles were 
expressed as the percentage of RVC. We calculated the ratios of muscle activation as the \% RVCs of IO relative to RA (IO/RA ratio), TrA relative to RA (TrA/RA ratio), and LM relative to LES (LM/LES ratio).

We measured the pain intensity using a visual analog scale (VAS) and evaluated the LBP-correlated functional disability using the Oswestry Disability Index (ODI). The pain and disability levels were assessed at T0 (initial), T1 (after four weeks), and T2 (after 12 weeks).

\section{Statistical analysis}

We performed all the statistical analyses with SPSS version 22.0 (SPSS Korea Data Solution Inc., Seoul, Korea). We used Wilcoxon's signed-ranks tests to compare the muscle thickness ratio, the sEMG data, the disability level, and the pain intensity of the subjects before and after training; and Mann-Whitney $U$ tests to identify any significant difference in the baseline data between the two groups. We also used a repeated-measures analysis of variance (rm-ANOVA) to determine the presence of a temporary interaction of the three different measures within each group. We presented the three epidemiologic and measured variables and two explanatory variables of symptom in a regression analysis of each group. Statistical significance was considered at $p<0.05$.

\section{Results}

A total of 56 patients (27 in the CSE group and 29 in the VSE group) completed the full exercise program and evaluation. The clinical and demographic data of the participants are summarized in Table 1. There was no statistically significant difference in the baseline data between the two groups $(p>0.05)$. After the exercise, the VAS and ODI showed statistically significant clinical improvement in both groups which were maintained after 8 weeks. There were no significant intergroup differences (Table 2).

Table 1

Patients' clinical and demographic characteristics $(n=56)$

\begin{tabular}{|llll|}
\hline & $\begin{array}{l}\text { CSE group } \\
(\mathbf{n = 2 7 )}\end{array}$ & $\begin{array}{l}\text { VSE group } \\
(\mathbf{n = 2 9 )}\end{array}$ & $p$ value \\
\hline Sex (male:female) & $17: 10$ & $17: 12$ & 0.23 \\
\hline Age, years, mean \pm SD & $38.7 \pm 8.1$ & $37.4 \pm 10.7$ & 0.82 \\
\hline BMI, $\mathrm{kg} / \mathrm{m}^{2}$, mean \pm SD & $21.5 \pm 2.3$ & $22.3 \pm 2.2$ & 0.43 \\
\hline Symptom duration, months, mean \pm SD & $12.9 \pm 7.0$ & $15.0 \pm 11.1$ & 0.19 \\
\hline Initial VAS & $2.9 \pm 1.3$ & $3.1 \pm 1.6$ & 0.75 \\
\hline Initial ODI & $13.2 \pm 11.6$ & $14.8 \pm 9.2$ & 0.38 \\
\hline $\begin{array}{l}\text { CSE, conventional stabilization exercise; VSE, vibration stabilization exercise; SD, standard deviation; } \\
\text { BMI, body mass index; VAS, visual analog scale; ODI, Oswestry disability index (\%) }\end{array}$ & \\
\hline
\end{tabular}


Table 2

Pain and disability data before versus after training

\begin{tabular}{|c|c|c|c|c|c|}
\hline & & TO & T1 & $\mathrm{T} 2$ & $p$ value \\
\hline \multirow[t]{2}{*}{ CSE group } & VAS & $2.9 \pm 1.3$ & $1.8 \pm 1.3$ & $1.7 \pm 1.2$ & $<0.001^{*}$ \\
\hline & ODI & $13.2 \pm 11.6$ & $11.2 \pm 7.1$ & $10.9 \pm 9.6$ & $0.040^{*}$ \\
\hline \multirow[t]{2}{*}{ VSE group } & VAS & $3.1 \pm 1.6$ & $1.8 \pm 1.5$ & $1.6 \pm 1.2$ & $<0.001^{\star}$ \\
\hline & ODI & $14.8 \pm 9.2$ & $10.2 \pm 8.8$ & $9.9 \pm 7.6$ & $<0.001^{\star}$ \\
\hline \multicolumn{6}{|c|}{${ }^{*} p<0.05$; statistically different. } \\
\hline \multicolumn{6}{|c|}{$\begin{array}{l}\text { T0, pre-therapeutic period; T1, just after therapy; T2, } 8 \text { weeks follow-up after therapy; CSE, } \\
\text { conventional stabilization exercise; VAS, visual analog scale; ODI, Oswestry disability index (\%); VSE, } \\
\text { vibration stabilization exercise }\end{array}$} \\
\hline
\end{tabular}

We calculated the thickness ratios (contracted/rest) of each muscle. In the CSE group, the thickness ratios of the left IO, the bilateral TrA, and the LM muscles were statistically different before and after the exercise. In the VSE group, we found a significant difference for the left EO, the bilateral IO, the TrA, and the LM (Fig. 2).

We also found that the activation of the right RA, the bilateral EO, IO, and LM was statistically increased in the CSE group. In the VSE group, we measured a significantly higher level of activation in the bilateral IO, TrA, and LM. We also found that the bilateral LM/LES ratios in the CSE group, and the bilateral IO/RA, TrA/RA, and LM/LES in the VSE group were significantly increased (Fig. 3).

We used a multiple linear regression model to analyze the factors influencing VAS and ODI efficacy. This was calculated using the following formula: [Efficacy = (post-value - pre-value) / pre-value]. The epidemiologic data and the measured ratio of muscle thickness and activity were the independent factors and the efficacy of VAS and ODI, the dependent factors. The age, the initial measures obtained with the VAS and ODI, as well as the TrA thickness and LM/LES ratios had a statistically significant impact on the VAS and ODI efficacy in the CSE group (Table 3). In the VSE group, we found that the age, the initial measures obtained with the VAS and ODI, the TrA and LM thickness ratio as well as the IO/RA, TrA/RA, and LM/LES ratios had a statistically significant impact (Table 4). 
Table 3

Multiple regression analysis of epidemiologic and measured data for efficacy of VAS and ODI in the CSE group

\begin{tabular}{|c|c|c|c|c|c|c|}
\hline \multirow{2}{*}{$\begin{array}{l}\text { Independent } \\
\text { variables }\end{array}$} & \multicolumn{3}{|l|}{ Efficacy of VAS } & \multicolumn{3}{|l|}{ Efficacy of ODI } \\
\hline & $\begin{array}{l}\text { Unstandardized } \\
\text { coefficient }\end{array}$ & $\begin{array}{l}p- \\
\text { value }\end{array}$ & $\begin{array}{l}\text { Adjusted } \\
\text { R square }\end{array}$ & $\begin{array}{l}\text { Unstandardized } \\
\text { coefficient }\end{array}$ & $\begin{array}{l}p- \\
\text { value }\end{array}$ & $\begin{array}{l}\text { Adjusted } \\
\text { R square }\end{array}$ \\
\hline Age & -0.012 & $\stackrel{<}{0.001^{*}}$ & \multirow{5}{*}{$\begin{array}{l}0.873 \\
(p< \\
0.001)\end{array}$} & -0.005 & $0.016^{\star}$ & \multirow{5}{*}{$\begin{array}{l}0.578 \\
(p< \\
0.001)\end{array}$} \\
\hline Initial VAS & -0.030 & $0.045^{\star}$ & & -0.044 & $0.011^{\star}$ & \\
\hline Initial ODI & -0.004 & $<_{0.001 *}$ & & - & - & \\
\hline $\begin{array}{l}\text { TrA thickness } \\
\text { ratio }\end{array}$ & - & - & & -0.053 & $0.005^{\star}$ & \\
\hline $\begin{array}{l}\text { LM/LES ratio of } \\
\text { muscle activity }\end{array}$ & - & - & & -0.004 & $0.034^{\star}$ & \\
\hline \multicolumn{7}{|c|}{${ }^{\star} p<0.05$; statistically significant coefficient } \\
\hline $\begin{array}{l}\text { CSE, conventiona } \\
\text { TrA, transversus a } \\
\text { muscle. }\end{array}$ & $\begin{array}{l}\text { bilization exer } \\
\text { minis muscle; }\end{array}$ & $\begin{array}{l}\text { VAS, vi } \\
\text { lumbar }\end{array}$ & $\begin{array}{l}\text { l analo } \\
\text { ultifidus }\end{array}$ & $\begin{array}{l}\text { e; ODI, Oswestr } \\
\text { le; LES, lumbar }\end{array}$ & $\begin{array}{l}\text { sability } \\
\text { ctor sp }\end{array}$ & idex (\%); \\
\hline
\end{tabular}


Table 4

Multiple regression analysis of epidemiologic and measured data for efficacy of VAS and ODI in the VSE group

\begin{tabular}{|c|c|c|c|c|c|c|}
\hline \multirow{2}{*}{$\begin{array}{l}\text { Independent } \\
\text { variables }\end{array}$} & \multicolumn{3}{|l|}{ Efficacy of VAS } & \multicolumn{3}{|l|}{ Efficacy of ODI } \\
\hline & $\begin{array}{l}\text { Unstandardized } \\
\text { coefficient }\end{array}$ & $\begin{array}{l}p- \\
\text { value }\end{array}$ & $\begin{array}{l}\text { Adjusted } \\
\text { R square }\end{array}$ & $\begin{array}{l}\text { Unstandardized } \\
\text { coefficient }\end{array}$ & $\begin{array}{l}p- \\
\text { value }\end{array}$ & $\begin{array}{l}\text { Adjusted } \\
\text { R square }\end{array}$ \\
\hline Age & -0.014 & $\stackrel{<}{0.001^{*}}$ & \multirow{8}{*}{$\begin{array}{l}0.941 \\
\\
(p< \\
0.001)\end{array}$} & -0.011 & $\dot{<} 0.001^{*}$ & \multirow{8}{*}{$\begin{array}{l}0.896 \\
(p< \\
0.001)\end{array}$} \\
\hline Initial VAS & -0.023 & $\hat{0}_{0.001 *}$ & & - & - & \\
\hline Initial ODI & -0.011 & $<.001 *$ & & - & - & \\
\hline $\begin{array}{l}\text { TrA thickness } \\
\text { ratio }\end{array}$ & - & - & & -0.115 & $0.042^{\star}$ & \\
\hline $\begin{array}{l}\text { LM thickness } \\
\text { ratio }\end{array}$ & -0.035 & $0.005^{\star}$ & & -0.111 & $0.048^{\star}$ & \\
\hline $\begin{array}{l}\text { IO/RA ratio of } \\
\text { muscle activity }\end{array}$ & -0.09 & $0.002^{\star}$ & & -0.122 & $0.039 *$ & \\
\hline $\begin{array}{l}\text { TrA/RA ratio of } \\
\text { muscle activity }\end{array}$ & -0.29 & $0.003^{\star}$ & & -0.054 & $0.005^{\star}$ & \\
\hline $\begin{array}{l}\text { LM/LES ratio of } \\
\text { muscle activity }\end{array}$ & -0.11 & $\hat{0}_{0.001 *}$ & & -0.029 & $0.029 *$ & \\
\hline \multicolumn{7}{|c|}{${ }^{\star} p<0.05$; statistically significant coefficient } \\
\hline
\end{tabular}

\section{Discussion}

Spinal stabilization exercises are a single application and are used as effective adjuvant therapy for patients with low back pain. An exercise protocol that includes stability exercises has been reported to help reduce the pain and disability of the patients with low back pain, and several studies on the impact of therapeutic exercise on patients with LBP have focused on stabilization through the reinforcement of trunk muscles such as the TrA and the LM. In particular, researchers stated that the coordinated activity of the deep paraspinal muscles played an important role in fine-tuning the intersegmental mobility of the spine (24). We considered the available data to design a proper and compatible 8-session exercise protocol over a period of four weeks.

Whole-body vibration (WBV) using specific frequencies is a treatment technique currently marketed for low back pain. It has been suggested that WBV can help improve low back pain by promoting a stretch 
reflex action and by strengthening trunk muscles, which are known to be weak in patients with low back pain $(25,26)$. Data also showed that WBV at frequencies below $20 \mathrm{~Hz}$ may reduce LBP by reducing spasms in the paravertebral muscles $(27,28)$. However, the method used for WBV relies on indirect vibrations applied to the lumbar part, which can cause perturbations on a systemic level.

Vibration therapy on muscles and tendons is administered by direct or indirect methods $(29,30)$. Indirect vibrations are usually applied using vibration platforms, dumbbells, and vibratory motors attached to resistance training machines and cables. On the other hand, direct vibrations are applied with electromotor devices that need a direct contact on the body. Focal vibrations on the musculoskeletal system can be applied to specific muscles or tendons and have tried to use for relief of focal spasticity due to upper motor neuron lesion $(31,32)$. However, there are few reports on the impact of strengthening and stabilizing the coordinated function of the trunk muscles on motor control by direct vibration.

Mischi et al. performed a meaningful study on indirect focal vibrations. The authors delivered $28 \mathrm{~Hz}$ vibration stimulation on the elbow flexor and extensor muscles during isometric contractions using a belt and found that this system caused a low-level co-contraction of the muscles (33). This was probably due to the increase of vibration-induced muscle activity and motor unit synchronization and the alteration of muscle spindle sensitivity. This mechanism resembles the well-studied tonic vibration reflex, which is a physiologic response to a mechanical vibration applied to muscles or tendons which can cause a reflex muscle contraction through la fibers (34). Based on this theory, some studies have reported that vibration applied at a certain frequency to limb muscles or tendons located at the extremities enhanced synchronization $(6,35)$. The study of Issurin et al. is noteworthy as it established the acute and residual effects of vibratory stimulation during explosive strength exercises. The vibration was applied focally on the biceps brachii muscle using an indirect method. The authors reported that the significant difference in the muscle response between elite and amateur athletes was associated with the higher sensitivity of the muscle receptors and the central nervous system of elite athletes due to additional stimulation (36). Core stabilization exercise using a sling with vibratory stimulation is similar to a direct method and it is based on regional vibration stimuli. The vibration generator is connected to the harness via a sling rope, which is in contact with a part of the body. The design of this system is also associated with the clinical efficacy of various vibration treatments based on the existing tonic vibration reflex (TVR) theory (37). In a comparative study using this system on healthy subjects, the authors reported a significant increase in the activity of trunk muscles (38).

To our knowledge, there has been no study on the effect of direct focal vibration on the contraction of trunk muscles. This was part of an effort to find an efficient core stabilization exercise method that any patients could do on their own. Sling exercises associated with vibration stimulation is effective and has good compliance, however, it is difficult to implement at home.

In addition, a comprehensive review of existing studies suggests that the TVR effect is influenced by muscular facilitation. In other words, TVR is expected to augment the motor stimulation in the contracted muscle and increase its force. Mischi et al. also reported a similar phenomenon and showed differences 
in the EMG activity of the elbow flexor and extensor muscles after upper extremity exercises with vibration stimulus. The researchers introduced this as an interesting observation and concluded that this was a preferred neural strategy for the more commonly used flexors in daily activities (33). Further studies will confirm whether the vibrational effects are selectively occurring during muscular contraction and relaxation.

This study has several limitations. Mostly, we could not control the variability of the physical vibration. To promote the compliance of the patients in the VSE group, we applied the vibrations in a relaxed state before initiating the treatment and set the intensity and frequency at a level acceptable for the subject. Therefore, the frequency and intensity applied to each subject were different. Further research should be carefully designed to control this variable and assess the physical quantities of vibration applied to the human body. Besides, we could not evaluate the sustainability of the vibration effect as self-exercise of the subjects was not controlled between T1 and T2. Additionally, we could not perform a reevaluation of the sEMG. This issue requires an independent research design.

\section{Conclusion}

In this study, we showed that direct and focal vibration stimulation during the contraction of the trunk muscles through stabilization exercises significantly changed the muscle activity pattern. This suggests important implications for future research. The precise control of vibrations and anatomical considerations are still needed to establish evidence for the effects of vibration on neurophysiological mechanisms.

\section{Abbreviations}

ADIM: abdominal drawing-in maneuver. CLBP: non-specific chronic low back pain, CSE: conventional stabilization exercise, VSE: vibration-plus stabilization exercise, EO: external oblique, IO: internal oblique, LES: lumbar erector spinae, LM: lumbar multifidus, ODI: Oswestry Disability Index, RA: rectus abdominis, RVC: Reference voluntary contraction, sEMG: surface electromyography, TrA: transverse abdominis, TVR: tonic vibration reflex, VAS: visual analogue scale, WBV: whole-body vibration

\section{Declarations}

\section{Acknowledgements}

This research was supported by a grant of Korea University Ansan Hospital (01903511) in 2019. The authors would also like to thank all of the participants for their commitment to the study.

\section{Authors' contribution}

NK contributed to the conception and design of the work; data acquisition, analysis and interpretation, work draft and revision; SHY contributed to the data acquisition, analysis and interpretation; MK 
contributed to the data acquisition, analysis and interpretation; SHL contributed to the data acquisition, analysis and interpretation; DHK contributed to the data acquisition, analysis and interpretation; KHK contributed to the data acquisition, analysis and interpretation; All authors have read and approved the manuscript.

\section{Availability of data and materials}

Data are available by contacting the corresponding author.

\section{Ethics approval and consent to participate}

Written informed consent was obtained from all subjects. The study was approved by the Institutional Review Board of Korea University Anam Hospital, Seoul, Korea (IRB No. 2018AN0361).

\section{Consent for publication}

Not applicable.

\section{Competing Interest}

The authors declare that they have no competing interests.

\section{References}

1. Standaert CJ, Weinstein SM, Rumpeltes J. Evidence-informed management of chronic low back pain with lumbar stabilization exercises. The spine journal : official journal of the North American Spine Society. 2008;8(1):114-20.

2. Koumantakis GA, Watson PJ, Oldham JA. Trunk muscle stabilization training plus general exercise versus general exercise only: randomized controlled trial of patients with recurrent low back pain. Physical therapy. 2005;85(3):209-25.

3. Niemisto L, Rissanen P, Sarna S, Lahtinen-Suopanki T, Lindgren KA, Hurri H. Cost-effectiveness of combined manipulation, stabilizing exercises, and physician consultation compared to physician consultation alone for chronic low back pain: a prospective randomized trial with 2-year follow-up. Spine. 2005;30(10):1109-15.

4. Rittweger J. Vibration as an exercise modality: how it may work, and what its potential might be. European journal of applied physiology. 2010;108(5):877-904.

5. Bosco C, Colli R, Introini E, Cardinale M, Tsarpela O, Madella A, et al. Adaptive responses of human skeletal muscle to vibration exposure. Clinical physiology (Oxford, England). 1999;19(2):183-7.

6. Bosco C, Cardinale M, Tsarpela O. Influence of vibration on mechanical power and electromyogram activity in human arm flexor muscles. European journal of applied physiology and occupational physiology. 1999;79(4):306-11. 
7. Mileva KN, Naleem AA, Biswas SK, Marwood S, Bowtell JL. Acute effects of a vibration-like stimulus during knee extension exercise. Medicine and science in sports and exercise. 2006;38(7):1317-28.

8. Bazett-Jones DM, Finch HW, Dugan EL. Comparing the effects of various whole-body vibration accelerations on counter-movement jump performance. Journal of sports science \& medicine. 2008;7(1):144-50.

9. Torabi M, Okhovatian F, Naimi SS, Baghban AA. Spinal Stabilization Exercise with and without Whole-Body Vibration: A Randomized Controlled Clinical Trial. Journal of Clinical Physiotherapy Research. 2016;1(2):86-90.

10. Luo J, McNamara B, Moran K. The use of vibration training to enhance muscle strength and power. Sports medicine (Auckland, NZ). 2005;35(1):23-41.

11. Koes BW, van Tulder M, Lin CW, Macedo LG, McAuley J, Maher C. An updated overview of clinical guidelines for the management of non-specific low back pain in primary care. European spine journal : official publication of the European Spine Society, the European Spinal Deformity Society, and the European Section of the Cervical Spine Research Society. 2010;19(12):2075-94.

12. Richardson C, Jull G, Hides J, Hodges P. Therapeutic exercise for spinal segmental stabilization in low back pain: Churchill Livingstone London; 1999.

13. O'Sullivan PB, Phyty GD, Twomey LT, Allison GT. Evaluation of specific stabilizing exercise in the treatment of chronic low back pain with radiologic diagnosis of spondylolysis or spondylolisthesis. Spine. 1997;22(24):2959-67.

14. O'Sullivan P, Twomey L, Allison G, Sinclair J, Miller K. Altered patterns of abdominal muscle activation in patients with chronic low back pain. The Australian journal of physiotherapy. 1997;43(2):91-8.

15. Teyhen DS, Gill NW, Whittaker JL, Henry SM, Hides JA, Hodges P. Rehabilitative ultrasound imaging of the abdominal muscles. The Journal of orthopaedic and sports physical therapy. 2007;37(8):45066.

16. Koppenhaver SL, Hebert JJ, Fritz JM, Parent EC, Teyhen DS, Magel JS. Reliability of rehabilitative ultrasound imaging of the transversus abdominis and lumbar multifidus muscles. Archives of physical medicine and rehabilitation. 2009;90(1):87-94.

17. Kiesel KB, Uhl TL, Underwood FB, Rodd DW, Nitz AJ. Measurement of lumbar multifidus muscle contraction with rehabilitative ultrasound imaging. Manual therapy. 2007;12(2):161-6.

18. Hermens HJ, Freriks B, Disselhorst-Klug C, Rau G. Development of recommendations for SEMG sensors and sensor placement procedures. J Electromyogr Kinesiol. 2000;10(5):361-74.

19. McGill SM. Electromyographic activity of the abdominal and low back musculature during the generation of isometric and dynamic axial trunk torque: implications for lumbar mechanics. Journal of orthopaedic research : official publication of the Orthopaedic Research Society. 1991;9(1):91-103.

20. de Seze MP, Cazalets JR. Anatomical optimization of skin electrode placement to record electromyographic activity of erector spinae muscles. Surgical and radiologic anatomy : SRA. 2008;30(2):137-43. 
21. Arokoski JP, Valta T, Airaksinen O, Kankaanpaa M. Back and abdominal muscle function during stabilization exercises. Archives of physical medicine and rehabilitation. 2001;82(8):1089-98.

22. Zipp P. Recommendations for the standardization of lead positions in surface electromyography. European journal of applied physiology and occupational physiology. 1982;50(1):41-54.

23. Hislop H. Daniels and Worthingham's Muscle Testing: Techniques of Manual Examination and Performance Testing, 9e: Elsevier India; 2013.

24. Hodges PW. Core stability exercise in chronic low back pain. The Orthopedic clinics of North America. 2003;34(2):245-54.

25. Hodges PW. Changes in motor planning of feedforward postural responses of the trunk muscles in low back pain. Experimental brain research. 2001;141(2):261-6.

26. Rittweger J, Just K, Kautzsch K, Reeg P, Felsenberg D. Treatment of chronic lower back pain with lumbar extension and whole-body vibration exercise: a randomized controlled trial. Spine. 2002;27(17):1829-34.

27. Fischer $\mathrm{AA}$, Chang $\mathrm{CH}$. Electromyographic evidence of paraspinal muscle spasm during sleep in patients with low back pain. The Clinical Journal of Pain. 1985;1(3):147-54.

28. Rittweger J, Mutschelknauss M, Felsenberg D. Acute changes in neuromuscular excitability after exhaustive whole body vibration exercise as compared to exhaustion by squatting exercise. Clinical physiology and functional imaging. 2003;23(2):81-6.

29. Brunetti O, Filippi GM, Lorenzini M, Liti A, Panichi R, Roscini M, et al. Improvement of posture stability by vibratory stimulation following anterior cruciate ligament reconstruction. Knee surgery, sports traumatology, arthroscopy : official journal of the ESSKA. 2006;14(11):1180-7.

30. Richardson MS, Cramer JT, Bemben DA, Shehab RL, Glover J, Bemben MG. Effects of age and ACL reconstruction on quadriceps gamma loop function. Journal of geriatric physical therapy (2001). 2006;29(1):28-34.

31. Caliandro P, Celletti C, Padua L, Minciotti I, Russo G, Granata G, et al. Focal muscle vibration in the treatment of upper limb spasticity: a pilot randomized controlled trial in patients with chronic stroke. Archives of physical medicine and rehabilitation. 2012;93(9):1656-61.

32. Camerota F, Celletti C, Di Sipio E, De Fino C, Simbolotti C, Germanotta M, et al. Focal muscle vibration, an effective rehabilitative approach in severe gait impairment due to multiple sclerosis. Journal of the neurological sciences. 2017;372:33-9.

33. Mischi $\mathrm{M}$, Cardinale $\mathrm{M}$. The effects of a $28-\mathrm{Hz}$ vibration on arm muscle activity during isometric exercise. Medicine and science in sports and exercise. 2009;41(3):645-53.

34. Hagbarth K, editor Motor effects of vibratory muscle stimuli in man. Noble Symposium I Muscular afferents and motor control; 1966: Almqvist \& Wiksell.

35. Martin BJ, Park HS. Analysis of the tonic vibration reflex: influence of vibration variables on motor unit synchronization and fatigue. European journal of applied physiology and occupational physiology. 1997;75(6):504-11. 
36. Issurin VB, Tenenbaum G. Acute and residual effects of vibratory stimulation on explosive strength in elite and amateur athletes. Journal of sports sciences. 1999;17(3):177-82.

37. Kirkesola G. Neurac-a new treatment method for long-term musculoskeletal pain. J Fysioterapeuten. 2009;76:16-25.

38. Kim JH, Kim YE, Bae SH, Kim KY. The effect of the neurac sling exercise on postural balance adjustment and muscular response patterns in chronic low back pain patients. Journal of physical therapy science. 2013;25(8):1015-9.

\section{Figures}

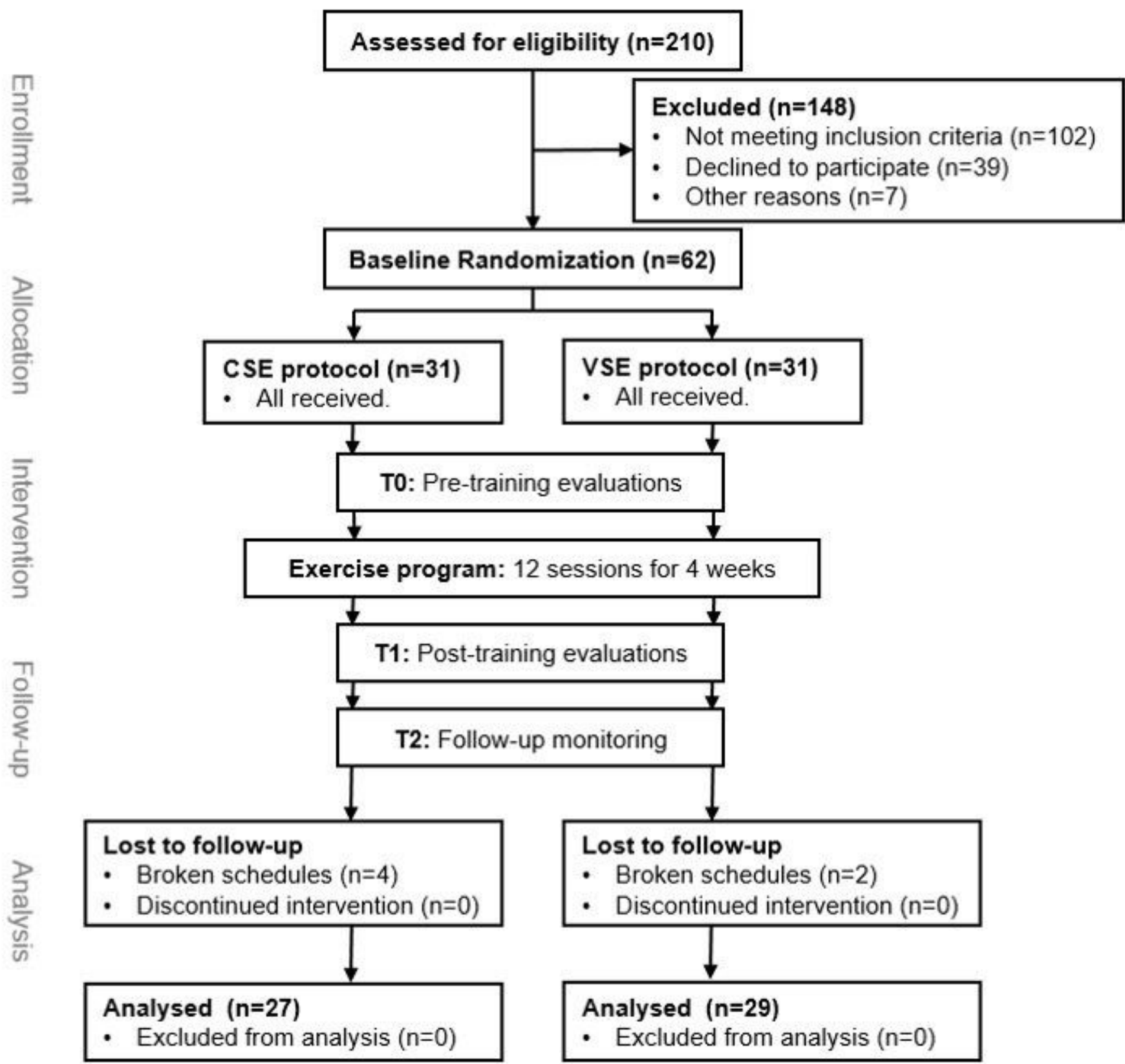

Figure 1 
Flow chart showing subject recruitment and randomization. $\mathrm{CLBP}=$ chronic low back pain; CSE = conventional stabilization exercise; VSE = vibration stabilization exercise; VAS = visual analog scale; ODI = Oswestry disability index; $\mathrm{sEMG} \mathrm{=} \mathrm{surface} \mathrm{electromyography.}$

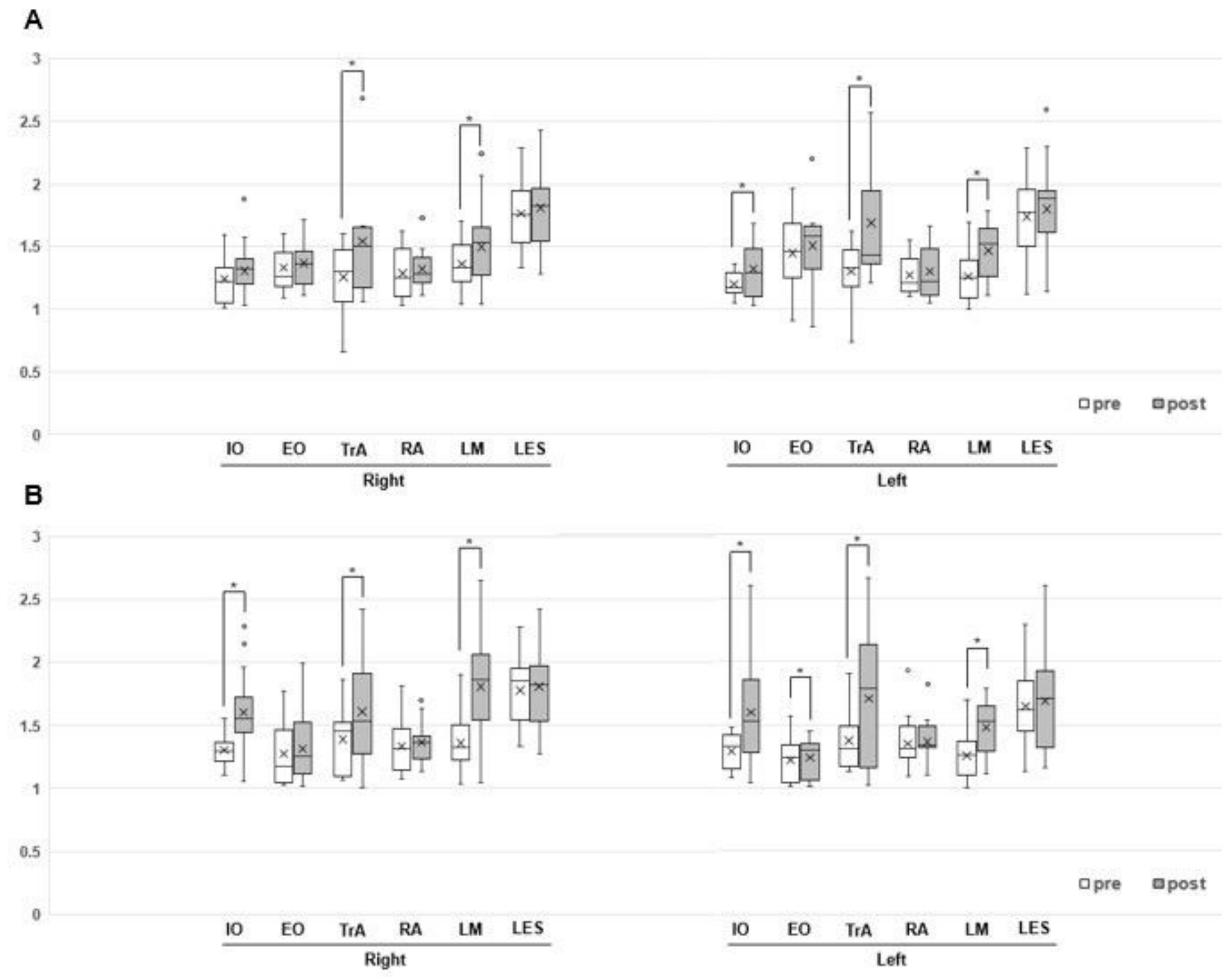

Figure 2

Ultrasonographic muscle thickness ratios (contracted/rest) for the trunk muscles of patients in the CSE (A) and the VSE (B) groups. * Statistical significance. TrA = transversus abdominis muscle; $10=$ internal oblique muscle; $\mathrm{EO}$ = external oblique muscle; $\mathrm{RA}$ = rectus abdominis muscle; $\mathrm{LM}=$ lumbar multifidus muscle; LES = lumbar erector spinae muscle 


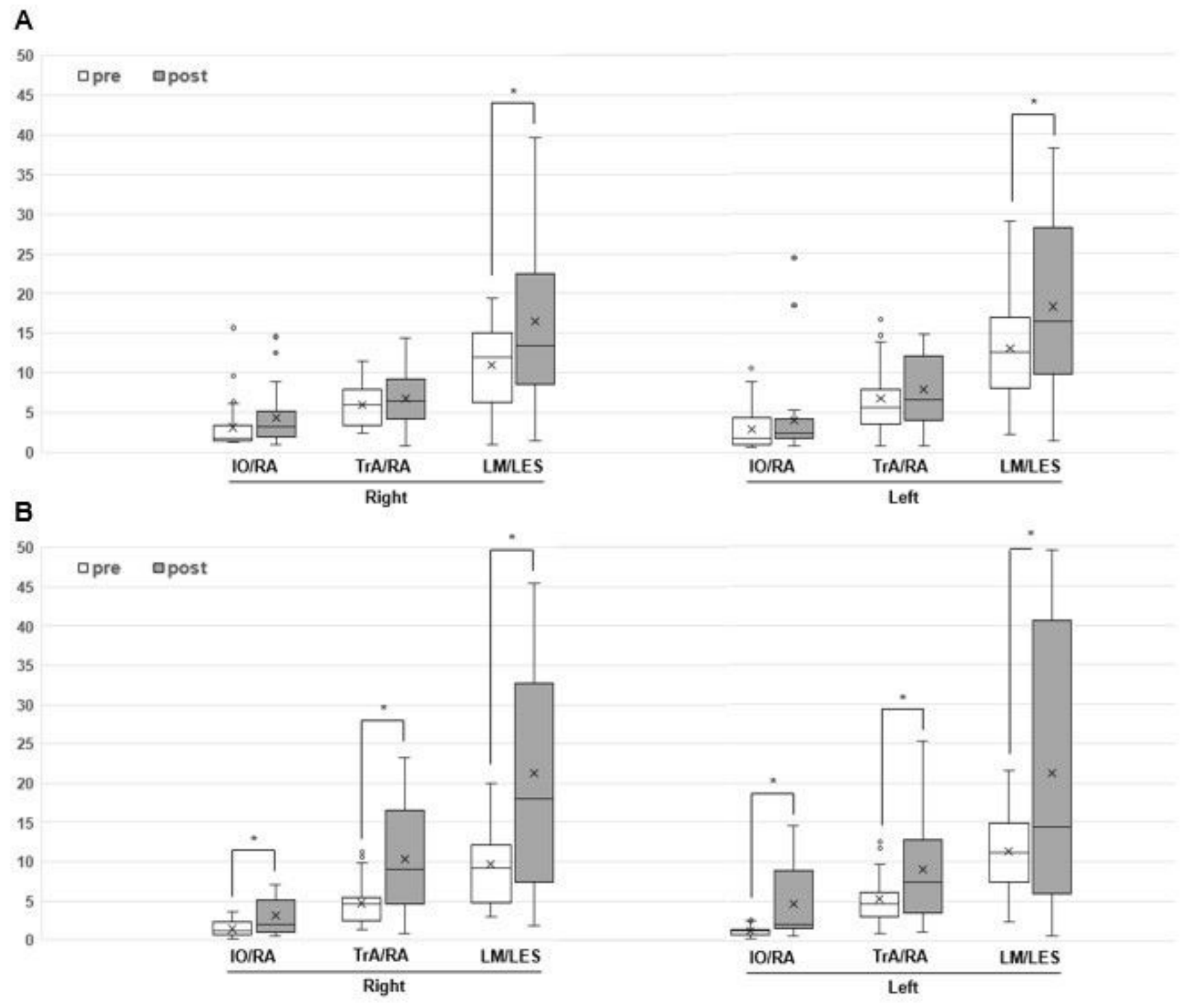

\section{Figure 3}

The ratio of muscle activation (IO/RA, TrA/RA and LM/LES muscle activities) in the CSE (A) and the VSE (B) groups. * Statistically significance. $\operatorname{Tr} A=$ transversus abdominis muscle; 10 = internal oblique muscle; $\mathrm{RA}=$ rectus abdominis muscle; $\mathrm{LM}=$ lumbar multifidus muscle; $\mathrm{LES}=$ lumbar erector spinae muscle.

\section{Supplementary Files}

This is a list of supplementary files associated with this preprint. Click to download.

- supplementary.xlsx 